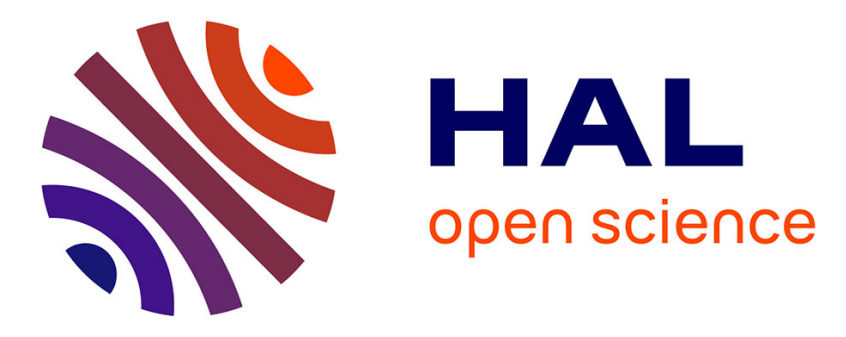

\title{
Characterization of mid-infrared femtosecond pulses [Invited]
}

Kevin F. Lee, Kevin J. Kubarych, Adeline Bonvalet, Manuel Joffre

\section{To cite this version:}

Kevin F. Lee, Kevin J. Kubarych, Adeline Bonvalet, Manuel Joffre. Characterization of mid-infrared femtosecond pulses [Invited]. Journal of the Optical Society of America B, 2008, 25 (6), pp.A54. 10.1364/JOSAB.25.000A54 . hal-00824264

\section{HAL Id: hal-00824264}

https://hal-polytechnique.archives-ouvertes.fr/hal-00824264

Submitted on 23 May 2014

HAL is a multi-disciplinary open access archive for the deposit and dissemination of scientific research documents, whether they are published or not. The documents may come from teaching and research institutions in France or abroad, or from public or private research centers.
L'archive ouverte pluridisciplinaire HAL, est destinée au dépôt et à la diffusion de documents scientifiques de niveau recherche, publiés ou non, émanant des établissements d'enseignement et de recherche français ou étrangers, des laboratoires publics ou privés. 


\title{
Characterization of mid-infrared femtosecond pulses [Invited]
}

\author{
Kevin F. Lee, ${ }^{1,2}$ Kevin J. Kubarych,, ${ }^{1,2,3}$ Adeline Bonvalet, ${ }^{1,2}$ and Manuel Joffre ${ }^{1,2, *}$ \\ ${ }^{1}$ Laboratoire d'Optique et Biosciences, Ecole Polytechnique, Centre National de la Recherche Scientifique, \\ 91128 Palaiseau, France \\ ${ }^{2}$ Institut National de la Santé et de la Recherche Médicale, U696, 91128 Palaiseau, France \\ ${ }^{3}$ Department of Chemistry, University of Michigan, Ann Arbor, Michigan 48109, USA \\ *Corresponding author: manuel.joffre@polytechnique.edu
}

Received November 15, 2007; revised February 4, 2008; accepted February 11, 2008; posted February 26, 2008 (Doc. ID 89830); published April 22, 2008

\begin{abstract}
We review different methods for characterizing mid-infrared femtosecond pulses, including linear methods such as electro-optic sampling, time- and frequency-domain interferometry, and nonlinear self-referenced methods such as frequency-resolved optical gating (FROG) and spectral phase interferometry for direct electric-field reconstruction (SPIDER). Of particular interest are methods based on upconversion through nonlinear mixing with chirped $800 \mathrm{~nm}$ pulses, enabling a complete measurement of mid-infrared pulses with visible-light spectrometers. (C) 2008 Optical Society of America
\end{abstract}

OCIS codes: $320.7100,120.5050$.

\section{INTRODUCTION}

Continuous progress in the generation, shaping, and characterization of mid-infrared (mid-IR) femtosecond pulses has driven new developments in IR femtosecond spectroscopy such as ultrafast vibrational pump-probe spectroscopy, vibrational coherent control, and IR multidimensional spectroscopy [1]. As with experiments in other spectral domains, interpretation of the results can depend strongly on the accurate characterization of the electric field as a function of time.

In general, the characterization of an ultrashort pulse consists of the complete measurement of the associated electric field, $E(t)$, or conversely of its Fourier transform [2], $E(\omega)=|E(\omega)| \exp (i \varphi(\omega))$. Since the measurement of the power spectrum is usually straightforward, the problem is often reduced to the measurement of the pulse spectral phase $\varphi(\omega)$. Note that the real quantity $E(t)$ is often written as the real part of the associated analytic signal [2], or complex field, $\mathcal{E}(t)=|\mathcal{E}(t)| \exp (i \phi(t))$ :

$$
E(t)=\operatorname{Re} \mathcal{E}(t)=\frac{\mathcal{E}(t)+\mathcal{E}^{*}(t)}{2} .
$$

By definition, the complex field contains only positivefrequency components, so that the relation between $\mathcal{E}$ and $E$ is straightforward in the frequency domain:

$$
\mathcal{E}(\omega)=2 \Theta(\omega) E(\omega),
$$

where $\Theta(\omega)$ stands for the Heaviside function. The entire transformation yielding the complex field $\mathcal{E}(t)$ from the real field $E(t)$ thus reads

$$
E(t) \stackrel{F . T .}{\rightarrow} E(\omega) \stackrel{\times 2 \Theta(\omega)}{\rightarrow} \mathcal{E}(\omega) \stackrel{F . T .^{-1}}{\rightarrow} \mathcal{E}(t),
$$

where $F . T$. stands for Fourier transform. As will be discussed later in this article, this simple processing through
Fourier space, illustrated in Fig. 1, is intimately related to numerical methods widely used for extracting the phase from interference patterns in space [3-5], frequency [6,7], or time [8] domains. Finally, we remark that, unless specified, the methods discussed below will not be sensitive to the carrier-envelope (CE) phase [9] so that it will be sufficient to measure the first-order derivative of the spectral phase, $\mathrm{d} \varphi / \mathrm{d} \omega$.

This article will discuss the characterization of mid-IR femtosecond pulses by a number of recently developed methods. We refer here to the mid-IR spectral domain as radiation of wavelengths between 3 and $20 \mu \mathrm{m}$. Since this spectral domain lies between the terahertz and the visible/near-IR spectral domains, many of the measurement methods we will discuss are direct adaptations of methods from these neighboring spectral domains where measurement technology is more advanced.

In adapting to the mid-IR, aspects specific to the mid-IR must also be taken into account. For example, the low energy of mid-IR photons means that sophisticated silicon-based charge-coupled device (CCD) detectors cannot be used directly with mid-IR light. Also, a mid-IR pulse is usually accompanied by visible pulses as a result of the nonlinear conversions used to produce mid-IR light. The type of pulses available often determines the most appropriate characterization method. Mid-IR generation might be done by difference-frequency mixing between the two tunable pulses from an optical parametric amplifier pumped by a Ti:sapphire chirped-pulsed amplifier (CPA) $[10,11]$. Another approach is the optical rectification of $10 \mathrm{fs}$ pulses, an extension of far-IR (or terahertz) techniques for generating single-cycle pulses in the midIR, and useful for both amplified and unamplified laser sources [12-16]. Although self-referenced methods can be used in the mid-IR, alternative characterization methods can take advantage of the other available pulses. 


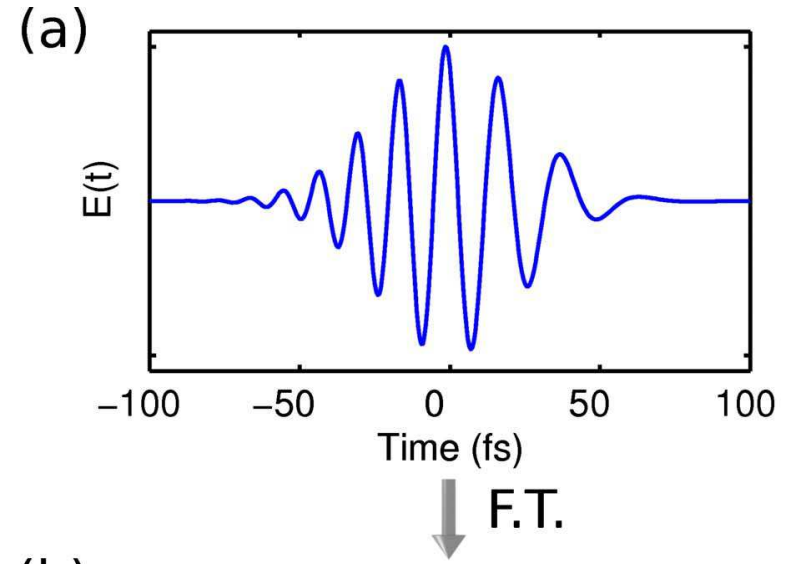

(b)
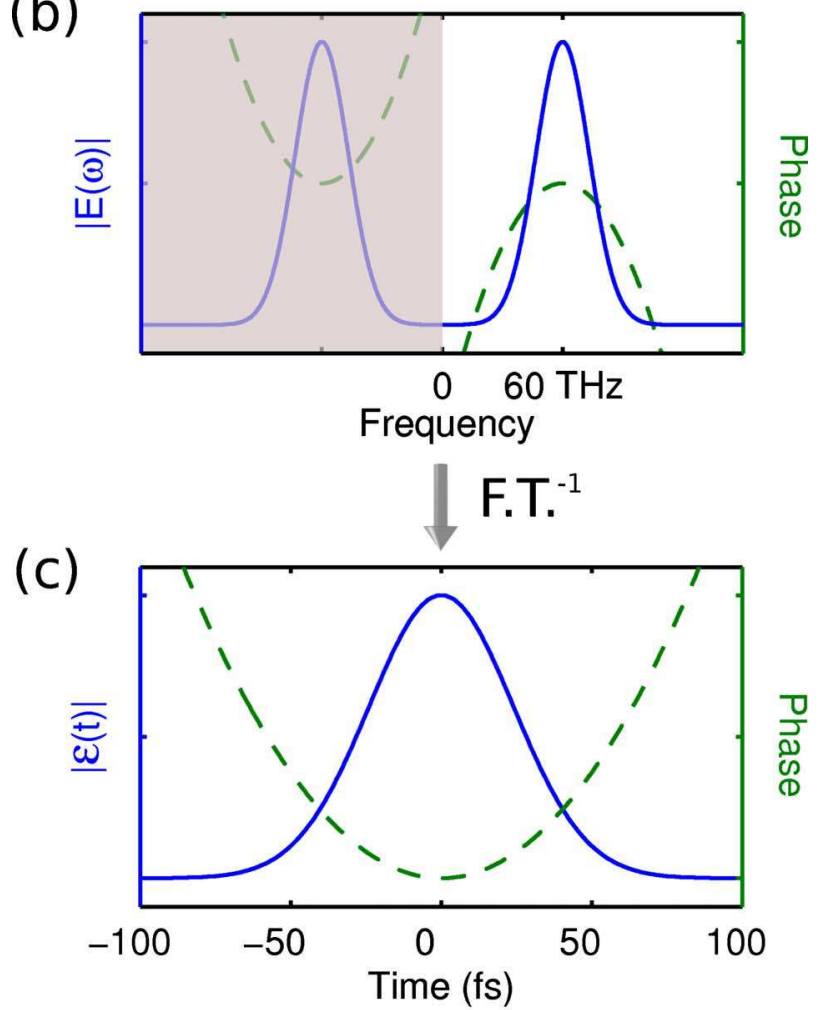

Fig. 1. (Color online) Illustration of the retrieval of the analytic signal $\mathcal{E}(t)$ from the real field $E(t)$ according to the recipe associated with expression (3). (a) Real electric field $E(t)$ in time domain in the case of a mid-IR chirped pulse centered at $5 \mu \mathrm{m}$. (b) Amplitude (-) and phase (- -) of $E(\omega)$ obtained from the real field through a Fourier transform (F.T.). The analytic signal $\mathcal{E}(\omega)$ is then obtained by zeroing out the negative-frequency components shown in the shaded area, according to Eq. (2). (c) Amplitude (-) and phase $(--)$ of the complex field $\mathcal{E}(t)$ obtained through an inverse Fourier transform of $\mathcal{E}(\omega)$. Note that the linear part $-\omega_{0} t$ of the time-domain phase $\phi(t)$ has been removed (where $\omega_{0}$ is the pulse center frequency).

In Section 2, we discuss measurement methods that are linear in the electric field, which are thus sensitive but require an appropriate reference pulse. In Section 3, we discuss self-referenced methods, which are less sensitive because they are based on a signal that is a fourthorder function of the electric field. Finally, in Section 4, we discuss a recently introduced upconversion method [17] that offers an especially convenient way to characterize mid-IR pulses produced by CPA laser systems.

\section{LINEAR MEASUREMENT METHODS}

In the case of radio waves, the oscillating electric field associated with an electromagnetic pulse can be directly measured using easily available electronic devices. Such devices are far too slow to acquire the rapidly oscillating electric field associated with optical and IR pulses. Hence, photodetectors generally do not respond directly to the electric field but to the pulse energy, which is quadratic in the electric field. However, detection schemes directly sensitive to the electric field can still be designed if one makes use of a correlation process involving an event shorter than the oscillation period of the field. This event can be a pulse of higher carrier frequency with a duration shorter than the oscillation period of the measured field, as in the electro-optic sampling method discussed in Subsection 2.A. One can also use another rapidly oscillating field whose frequency spectrum encompasses that of the measured field, as in the interferometric methods discussed in Subsection 2.B.

\section{A. Free-Space Electro-Optic Sampling}

Correlation methods for directly sampling an electric field were first developed for terahertz pulses. The oscillations of terahertz pulses are slow enough to be sampled by $100 \mathrm{fs}$ visible pulses by using either photoconductive switching [18] or electro-optic sampling [19]. The electrooptic sampling method has been extended to the mid-IR spectral domain by using $10 \mathrm{fs}$ pulses [13,15,20-22]. A sampling approach, based on completely different physical processes, has even been demonstrated in the visible spectral domain for measuring $5 \mathrm{fs}$ pulses by use of 250 attosecond extreme ultraviolet (XUV) pulses [23].

Figure 2(a) shows a typical setup for the free-space electro-optic sampling of mid-IR pulses. Both the mid-IR pulse and a visible pulse of typically $10 \mathrm{fs}$ duration are focused on a quadratic nonlinear crystal such as ZnTe, GaP, or GaSe. Through the electro-optic effect, the mid-IR pulse induces a birefringence in the crystal, which results in a change in the polarization state of the visible pulse. The differential signal detected after a Wollaston prism is then roughly proportional to the mid-IR field at the time of overlap between the two pulses. This sampling time can be varied by scanning the time delay $\tau$ between the two pulses in order to retrieve the variation of the mid-IR electric field as a function of time. The measured signal $S(\tau)$ is actually not exactly proportional to $E(\tau)$ because the sampling is not instantaneous. However, $S(\tau)$ is a linear and time-invariant function of the electric field. It is thus the convolution, $R(t) \otimes E(t)$, of the apparatus response function and the mid-IR field:

$$
S(\tau)=\int_{-\infty}^{+\infty} R(\tau-t) E(t) \mathrm{d} t=\int_{-\infty}^{+\infty} R(\omega) E(\omega) \exp (-i \omega \tau) \frac{\mathrm{d} \omega}{2 \pi},
$$

where $R(\omega)$ is the frequency-domain response function. If $R(\omega)$ is carefully simulated by taking into account the finite duration of the visible pulse and the frequency dependence of the phase-matching process, a complete retrieval of the electric field $E(t)$ can be performed through a straightforward deconvolution procedure [20,22]. Note 

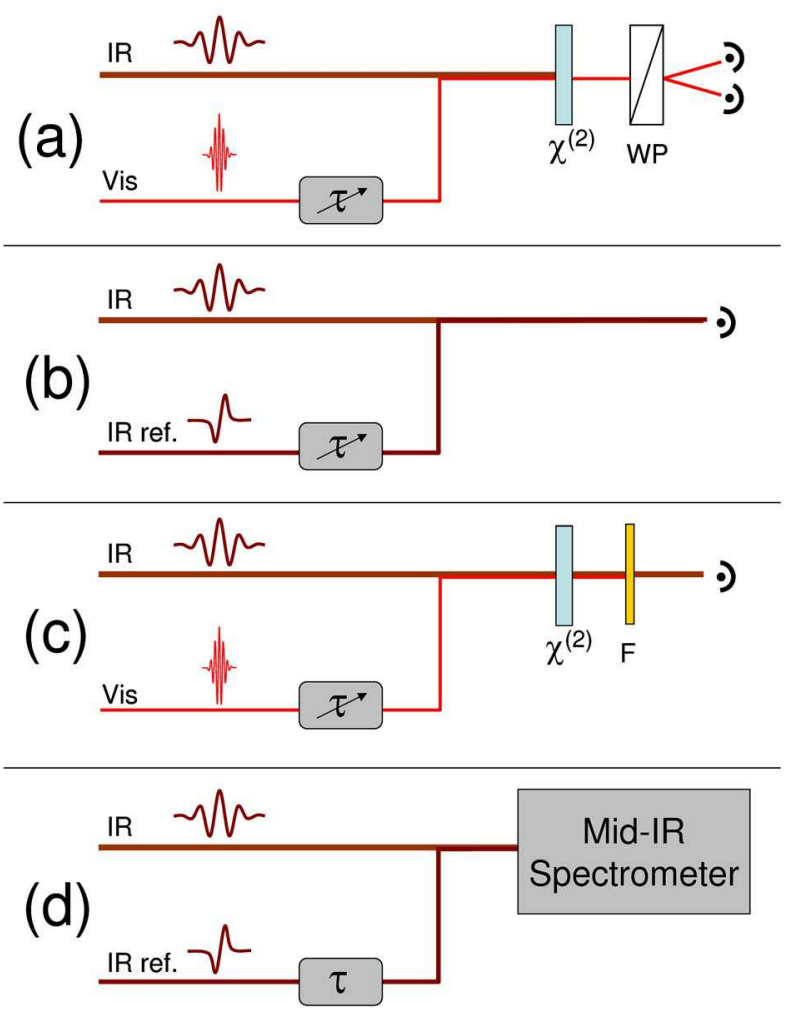

Fig. 2. (Color online) Linear methods for characterizing mid-IR femtosecond pulses. (a) Electro-sampling technique using a reference ultrashort visible pulse. WP stands for Wollaston prism. The slanted arrows indicate a scanned variable. (b) Time-domain interferometry using a reference ultrashort mid-IR pulse. (c) Time-domain interferometry using a reference visible ultrashort pulse and optical rectification. (d) Spectral interferometry using a reference ultrashort mid-IR pulse.

that electro-optic sampling is sensitive to the CE phase, so it is suitable only for pulses with a stable CE phase, which turns out to be the case for mid-IR pulses generated through optical rectification.

\section{B. Time- and Frequency-Domain Interferometry}

When a reference mid-IR pulse is available, the mid-IR field $E(t)$ can also be sampled through interferometric methods, for example, in the time domain as shown in Fig. 2(b). Let us call $E_{0}(t)$ the electric field associated with the reference pulse.

The total energy collected by the time-integrating mid-IR detector then reads

$$
\begin{aligned}
S(\tau) & =\int_{-\infty}^{+\infty}\left[E_{0}(t-\tau)+E(t)\right]^{2} \mathrm{~d} t \\
& =\text { Constant }+2 \int_{-\infty}^{+\infty} E_{0}(t-\tau) E(t) \mathrm{d} t .
\end{aligned}
$$

The signal $S(\tau)$ recorded as a function of the time delay $\tau$ thus yields the correlation function, $E_{0}(-t) \otimes E(t)$, between the two fields. Its Fourier transform is equal to $E_{0}^{*}(\omega) E(\omega)$ and provides the complex field $E(\omega)$ as long as the reference field $E_{0}(\omega)$ is fully characterized and its spectrum encompasses that of the measured field. Note that when the reference pulse is significantly shorter than the measured field, the deconvolution procedure described above is unnecessary.

Time-domain mid-IR interferometry can also be implemented as shown in Fig. 2(c), where the mid-IR beam is first recombined with a visible beam and then focused in a nonlinear crystal. The visible pulse is then converted into a mid-IR reference pulse and can interfere with the transmitted mid-IR field on a time-integrating mid-IR detector $[24,25]$. Interestingly, this implementation is closely related to that of electro-optic sampling shown in Fig. 2(a), especially if we recognize that optical rectification is also known as the inverse electro-optic effect. Indeed, since the setup preceding the nonlinear crystal is identical, the two methods only differ in the way the signal is measured. Time-domain interferometry for measuring mid-IR pulses has been applied, for example, to the measurement of the coherent mid-IR emission from a quantum-well structure [26] to multidimensional IR spectroscopy $[24,27]$ and to the measurement of shaped mid-IR pulses $[25,28,29]$.

The time-consuming process of scanning the time delay $\tau$ as well as the requirement for accurate phase control during the scanning can be avoided by using frequencydomain interferometry [6,30,31]. The setup, shown in Fig. $2(\mathrm{~d})$, is identical to that of time-domain interferometry except that the integrating detector is replaced with a mid-IR spectrometer while the time delay $\tau$ is kept constant. The measured signal reads

$$
S(\omega)=\left|E(\omega)+E_{0}(\omega) \exp (i \omega \tau)\right|^{2} .
$$

For a suitable choice of the time delay $\tau$, the measured spectrum exhibits spectral fringes that encode the phase difference between the two fields. The phase can be extracted by use of a Fourier-processing method similar to that illustrated in Fig. 1, except that the roles of $t$ and $\omega$ should be exchanged, and the window function in Fourier space must be slightly narrower than the Heaviside function used in expression (3). More precisely, the Fourier transform of the total spectrum is the linear autocorrelation of the total electric field, $E_{0}(t-\tau)+E(t)$, which includes the linear correlation $E_{0}(-t) \otimes E(t)$ between the two fields. In the time domain, the contribution from the correlation term separates from the other terms and can be easily isolated, yielding the same information as timedomain interferometry. An inverse Fourier transform then yields the product between the two fields in the frequency domain $[3,6]$.

Spectral interferometry is routinely used in IR multidimensional spectroscopy [27,32-34]. Multidimensional spectroscopy significantly benefits from a frequencymultiplexed spectrometer. At a fundamental level, dispersive spectrometers have a frequency-dependent spectral resolution for a given diffraction grating groove density, whereas a Fourier transform spectrum has uniform resolution across the entire spectrum. IR-sensitive detectors are available only in low-pixel-count arrays of 64 to 128 pixels-a sharp contrast to the megapixel CCD cameras in routine use. Since the intrinsic detectivity of silicon in the visible is two orders of magnitude greater than that of $\mathrm{HgCdTe}$ in the IR, even a $0.1 \%$ efficient upconversion process will be competitive with direct mid-IR detection. 


\section{SELF-REFERENCED NONLINEAR METHODS}

It is well known that stationary self-referenced methods for measuring ultrashort pulses require the use of nonlinear optical processes $[6,35,36]$. The measurement of mid-IR pulses is no exception to this general rule and usually relies on direct transpositions of methods previously developed for measuring visible pulses, such as second-order interferometric autocorrelation [37], secondharmonic generation frequency-resolved optical gating (SHG FROG) [38], and spectral phase interferometry for direct electric-field reconstruction (SPIDER) [7]. All of these methods rely on a second-order nonlinear process [39], followed by a time-integrating detector quadratic in the electric field, so that the final signal is a fourth-order function of the electric field.

\section{A. Second-Order Autocorrelation}

As shown in Fig. 3(a), a second-order interferometric autocorrelator consists of an interferometer producing two replicas of the incident pulse separated by a variable time delay $\tau$. SHG in a nonlinear crystal produces a frequency-
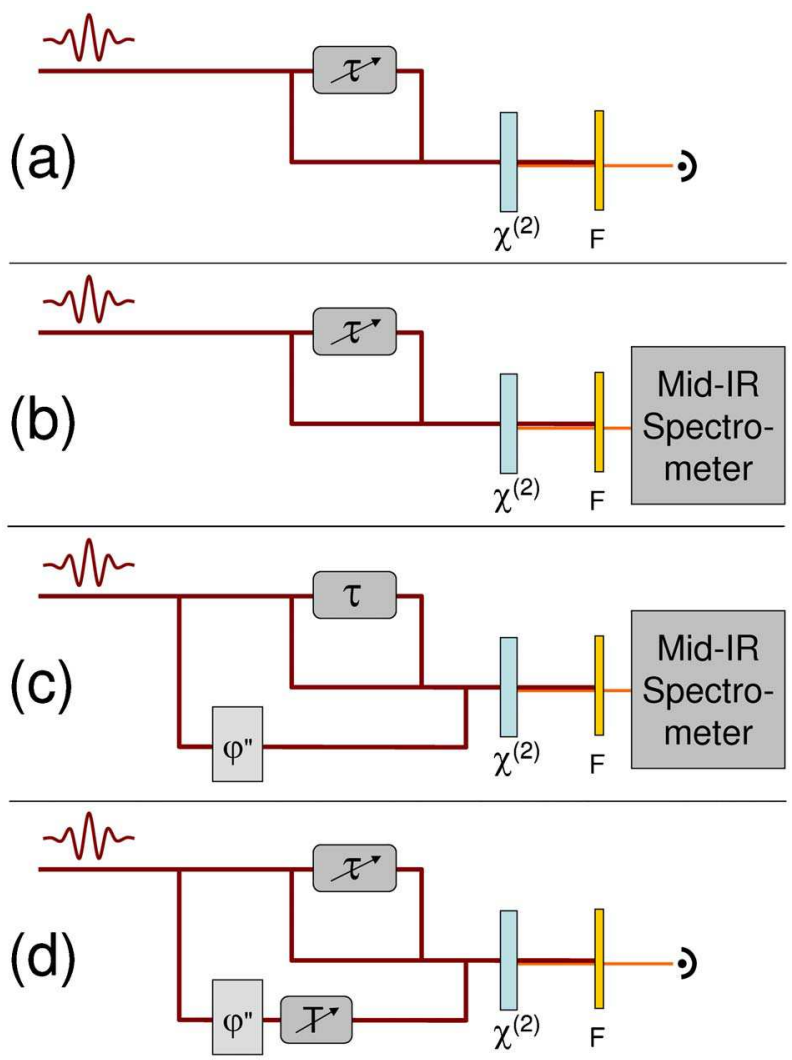

Fig. 3. (Color online) Self-referenced nonlinear methods for measuring a mid-IR femtosecond pulse. All the methods shown are based on sum-frequency mixing using the second-order susceptibility $\chi^{(2)}$ of a suitable nonlinear material such as GaAs, $\mathrm{AgGaS}_{2}$, or $\mathrm{AgGaSe}_{2}$. A filter $\mathrm{F}$ rejects the incoming light at the fundamental frequency. (a) Nonlinear second-order autocorrelation. (b) Frequency-resolved optical gating (FROG). (c) Spectral phase interferometry for direct electric-field reconstruction (SPIDER). $\varphi$ " stands for a transparent material such as $\mathrm{CaF}_{2}$ introducing a suitable amount of second-order spectral phase. (d) Time-domain homodyne optical technique for SPIDER (HOT SPIDER). doubled field proportional to $[\mathcal{E}(t)+\mathcal{E}(t-\tau)]^{2}$, which is further squared during the quadratic photodetection process. The time-integrating detector then produces the signal

$$
S(\tau)=\int_{-\infty}^{+\infty}|\mathcal{E}(t)+\mathcal{E}(t-\tau)|^{4} \mathrm{~d} t
$$

known as the second-order interferometric autocorrelation function of the pulse. This method has been implemented in the mid-IR, e.g., for characterizing $40 \mathrm{fs}$ pulses centered at $3.7 \mu \mathrm{m}$ [40], $54 \mathrm{fs}$ pulses centered at $5.5 \mu \mathrm{m}$ [10], or $105 \mathrm{fs}$ pulses centered at $9.2 \mu \mathrm{m}$ [11,41], corresponding in all cases to FWHM durations of only three optical cycles. As for visible autocorrelators, a noncollinear geometry is sometimes used, thus avoiding interference effects and directly producing the background-free intensity autocorrelation [10]. However, this approach is associated with a loss in time resolution that is not negligible for few-cycle pulses; thus it is preferable to retrieve the intensity autocorrelation from the experimental interferometric autocorrelation through Fourier filtering. Finally, as for visible pulses, the nonlinear process can occur as two-photon absorption in the detector itself, as was demonstrated for $9.6 \mu \mathrm{m}$ pulses using an InSb photodetector [10].

Transform-limited pulses can be entirely characterized from the agreement between the experimental autocorrelation and that calculated from the experimental spectrum assuming a flat spectral phase. When a pulse is not transform limited, more elaborate methods such as FROG or SPIDER are preferred for retrieving the spectral phase.

\section{B. SHG FROG}

SHG FROG is based on the measurement of a spectrally resolved second-order autocorrelation $[38,42]$. The experimental setup, shown in Fig. 3(b), is the same as that of a conventional autocorrelator but with a spectrometer replacing the integrating detector. In its standard form, SHG FROG uses a noncollinear geometry so that only the cross term $\mathcal{E}(t) \mathcal{E}(t-\tau)$ contributes to the collected SHG field. The corresponding spectrum detected by the spectrometer reads

$$
S(\tau, \omega)=\left|\int_{-\infty}^{+\infty} \mathcal{E}(t) \mathcal{E}(t-\tau) \exp (i \omega t) \mathrm{d} t\right|^{2},
$$

where $\omega$ is the detected frequency. The two-dimensional data set $S(\tau, \omega)$ is called the FROG trace and contains enough information to retrieve the pulse spectral phase using an iterative algorithm [38]. An interferometric version of SHG FROG is also available [43,44], based on an interferometric autocorrelator with the advantage of collinearity mentioned above, which is an important feature for the few-cycle pulses routinely generated in the mid-IR.

SHG FROG has been demonstrated in the mid-IR for characterizing $1.6 \mathrm{ps}$ pulses centered at $5 \mu \mathrm{m}$ and produced by a free-electron laser [45]. This first demonstration used a scanning spectrometer and a single HgCdTe detector, but SHG FROG can also be implemented using InGaAs or HgCdTe detector arrays, as was shown, for example, in the 3-5 $\mu \mathrm{m}$ band [46]. Since the cost of such de- 
tectors is quite high, FROG variants based on a singlechannel detector [47] are still important alternatives for characterizing mid-IR pulses [48].

Other interesting variants of FROG have also been demonstrated, in the mid-IR, such as frequency-resolved pump-probe [49], based on third-order rather than second-order optical nonlinearities, and cross-correlation FROG methods [50-52].

\section{SPIDER}

SPIDER is a transposition of shearing interferometry to the frequency domain [7]. The spectral shear is usually achieved through nonlinear mixing between the measured field $\mathcal{E}(t)$ and a linearly chirped pulse, whose duration is long enough so that the variation of its instantaneous frequency can be considered negligible over the time range where $\mathcal{E}(t)$ is nonzero. $\mathcal{E}(t)$ is thus being mixed with a quasi-monochromatic field of frequency $\Omega_{1}$, and the generated field $\mathcal{E}\left(\omega-\Omega_{1}\right)$ is simply a translated version in frequency space of the original field. This process is repeated for a second replica of the measured field, except that its delay with respect to the chirped pulse is different so that it is shifted in frequency by a different amount, $\Omega_{2}$. These two fields are then arranged so as to produce interference fringes, either in the frequency [7], space [4,5], or time domains [8], and the resulting signal is spectrally resolved using a spectrometer. Finally, the complex quantity $\mathcal{E}^{*}\left(\omega-\Omega_{1}\right) \mathcal{E}\left(\omega-\Omega_{2}\right)$ can be retrieved by processing within the appropriate Fourier space $[3,6]$. Its phase reads

$$
\varphi\left(\omega-\Omega_{2}\right)-\varphi\left(\omega-\Omega_{1}\right) \approx \Omega \frac{\mathrm{d} \varphi}{\mathrm{d} \omega}
$$

where $\Omega=\Omega_{1}-\Omega_{2}$ is the shear frequency. The measurement thus yields the frequency dependence of the group delay. As shown in Fig. 3(c), conventional SPIDER is a self-referenced method where the chirped pulse is generated by stretching the incident pulse using either a grating compressor or linear dispersion in a transparent material. In the visible, the latter method-obviously more convenient_can be used only for pulses of sufficiently broad bandwidth corresponding to very short durations (10 fs or less). In contrast, mid-IR pulses are much easier to stretch due to the greater group velocity dispersion (GVD) of materials in this spectral domain.

Despite this advantage of easier dispersion management in the mid-IR, conventional SPIDER has, to our knowledge, not been implemented in the mid-IR yet, probably due to the fact that detector arrays are not as easily available in the mid-IR as in the visible. However, this difficulty has been overcome through the development of SPIDER variants that do not require a mid-IR detector array, such as non-self-referenced SPIDER methods, which will be discussed in Section 4, and a SPIDER method based on time-domain interferometry, which we now discuss.

A widely used and efficient method for measuring IR spectra without a grating spectrometer is Fouriertransform spectroscopy [53], which is based on the fact that the spectrum is defined as the Fourier transform of the field autocorrelation function. This approach can be easily applied to the complete characterization of a mid-IR pulse by noting that the Fourier transform of the second-order interferometric autocorrelation function includes the spectrum of the frequency-doubled pulse [37] and hence acts as a Fourier-transform spectrometer. Indeed, homodyne optical technique for SPIDER (HOT SPIDER) [54] can be readily implemented by use of timedomain interferometry $[11,41,55]$. The experimental setup associated with time-domain HOT SPIDER is shown in Fig. 3(d) and consists of an interferometric autocorrelator with an additional arm corresponding to the chirped pulse. A sequence of three correlation measurements, associated with three different values of the time delay $T$ with respect to the chirped pulse, enables the acquisition of the same information as in conventional HOT SPIDER [11,41]. Furthermore, this time-domain implementation of HOT SPIDER provides redundancy, as the experimental setup enables the simultaneous acquisition of the second-order interferometric autocorrelation. The consistency between the measured autocorrelation and the spectral phase retrieved from HOT SPIDER has been verified for both nearly transform-limited pulses and chirped pulses [41].

\section{CHARACTERIZING MID-IR PULSES WITH A VISIBLE SPECTROMETER}

Although self-referenced characterization devices have many advantages, they suffer from a lower sensitivity due to the fact that the measured signal is at least a fourthorder function of the electric field. The SPIDER variant known as modified SPIDER [56] addresses this point by using an independent and intense chirped pulse rather than generating it from the measured field. For characterizing mid-IR pulses, this approach has the additional, and more important, benefit that the upconverted field can be centered at a frequency much greater than twice the fundamental frequency. By using a small part of the uncompressed $800 \mathrm{~nm}$ chirped pulse available in any CPA laser system, it is then possible to translate the mid-IR pulse into the visible spectral domain, where it can be readily detected by a standard CCD-based spectrometer. We discuss two implementations of this chirped-pulse upconversion (CPU) scheme. Both methods use two replicas of the $800 \mathrm{~nm}$ chirped pulses, either in a noncollinear [17] or a collinear geometry.

\section{A. Zero-Additional-Phase SPIDER}

While conventional SPIDER uses two replicas of the measured pulse and a single chirped pulse, the variant known as zero-additional-phase (ZAP) SPIDER, originally developed for characterizing sub 20 fs pulses [57-59], directly mixes the measured pulse with two replicas of the chirped pulse. One side effect of this approach is that the two upconverted pulses are generated simultaneously, and thus lack the time delay required for the Fourier transform retrieval algorithm. As shown in Fig. 4, the ZAP SPIDER scheme addresses this point by using a noncollinear geometry between the two chirped pulses so that the two upconverted pulses can be collected independently and recombined collinearly with a suitable time delay. 


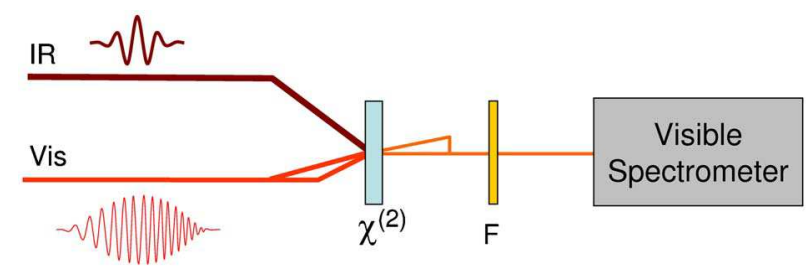

Fig. 4. (Color online) Characterization of mid-IR pulses using chirped-pulse upconversion (CPU) ZAP SPIDER.

This method has been recently demonstrated [17] for characterizing the amplitude and phase of mid-IR femtosecond pulses centered at $5.1 \mu \mathrm{m}$, upconverted in $\mathrm{MgO}: \mathrm{LiNbO}_{3}$, and detected around $695 \mathrm{~nm}$ using a 1340 $\times 100$ pixel CCD camera (Roper Scientific). The chirped pulse was the previously unused zero-order diffraction from the compressor grating of the CPA laser system (Hurricane, SpectraPhysics). Apart from the advantage, mentioned above, of detecting visible rather than mid-IR photons, this SPIDER implementation is also simpler to implement than the conventional variant [Fig. 3(c)], because the mid-IR part of the setup simply consists of focusing the mid-IR beam onto the nonlinear crystal. Furthermore, this method can be efficiently integrated into multidimensional [60] or pump-probe [61] experiments. After characterizing the local-oscillator or probe pulse in situ, the experiment can proceed by blocking one of the chirped $800 \mathrm{~nm}$ pulses, which turns the setup into an upconversion mid-IR spectrometer [17,62,63].

\section{B. Time-Encoded Arrangement SPIDER}

As mentioned in Subsection 3.C, rather than using frequency, some SPIDER variants employ either space [4,5] or time delay [8] to encode the phase in interference fringes. In this section, we discuss the mid-IR CPU implementation of the time-domain version, known as twodimensional spectral shearing interferometry (2DSI) [8]. In the following, we will refer to 2DSI as time-encoded arrangement (TEA) SPIDER, in order to avoid confusion with other 2D shearing methods such as spatially encoded arrangement (SEA) SPIDER [4,5]. As shown in Fig. 5 , the CPU implementation of TEA SPIDER is very simple. A TEA SPIDER setup is the same as a spectrally resolved CPU experiment, with the addition of an interferometer to the $800 \mathrm{~nm}$ path to create two chirped $800 \mathrm{~nm}$ pulses with a controlled time delay. In exchange for the simpler optical design, measuring the spectral phase requires the acquisition of a set of spectra for varying $800 \mathrm{~nm}$ pulse delay rather than a single spectrum.

In our experiment, the spectra are acquired individually at the $1 \mathrm{kHz}$ laser repetition rate using a $1340 \times 100$ pixel, front-illuminated CCD camera with 16 bit, $2 \mathrm{MHz}$ analog-to-digital conversion (Roper Scientific). To acquire

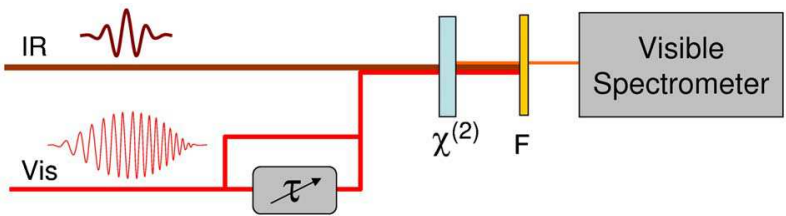

Fig. 5. (Color online) Characterization of mid-IR pulses using a time-encoded arrangement for SPIDER.

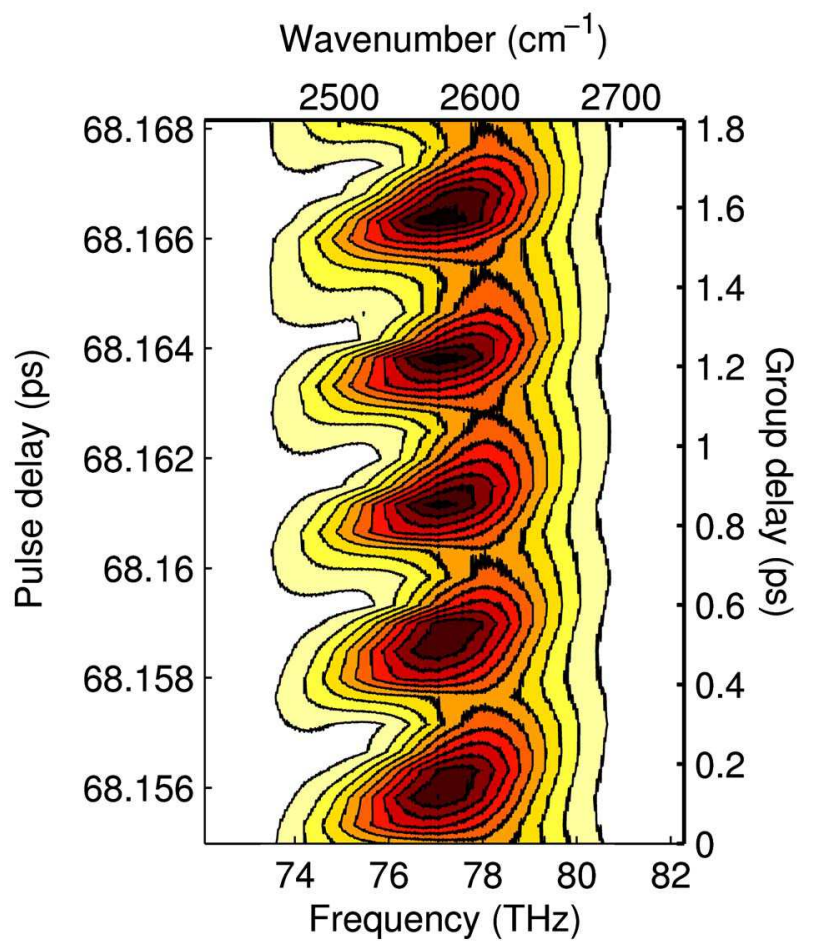

Fig. 6. (Color online) TEA SPIDER experimental trace associated with 31 consecutive laser shots for a chirped mid-IR femtosecond pulse. The $68 \mathrm{ps}$ delay between the two chirped $800 \mathrm{~nm}$ pulses corresponds to a shear frequency of $2.8 \mathrm{THz}$. This large value was chosen to make the tilt of the fringes more visible.

spectra at $1 \mathrm{kHz}$, we limited the active chip to 20 lines, which were vertically binned and packed into virtual images of $1340 \times 100$ pixels corresponding to 100 consecutive laser shots using a custom Labview driver (SITK for Labview, R Cubed). The position of the translation stage (Lot Oriel) was simultaneously recorded for each laser shot by monitoring the dc-motor quadrature encoder with a counter. Figure 6 shows a series of spectra obtained in the case of a mid-IR pulse of energy $1 \mu \mathrm{J}$, centered at $3.9 \mu \mathrm{m}$ and intentionally chirped through dispersion in a $2 \mathrm{~cm}$ $\mathrm{CaF}_{2}$ window. The $800 \mathrm{~nm}$ pulse energy was $0.1 \mathrm{~mJ}$ and the second-order derivative of its spectral phase was $-4 \mathrm{ps}^{2}$. Before calculation, the tilt of the fringes in the raw TEA SPIDER spectra already gives a good indication of the frequency dependence of the group delay [8]. The twodimensional phase $\Phi(\omega, \tau)$ of the TEA SPIDER trace reads

$$
\Phi(\omega, \tau)=\varphi\left(\omega-\Omega_{2}\right)-\varphi\left(\omega-\Omega_{1}\right)-\Omega_{1} \tau \approx \Omega \frac{\mathrm{d} \varphi}{\mathrm{d} \omega}-\Omega_{1} \tau
$$

where we use the same notation as in Subsection 3.C. $\Omega_{1}$ corresponds to the instantaneous frequency of the chirped pulse travelling through the moving arm of the interferometer. By noting that a fringe corresponds to a constant value of $\Phi$ and by isolating the group delay in the above equation, we can get the group delay by multiplying the delay $\tau$ by $\Omega_{1} / \Omega$, as was done for the right-side axis of Fig. 6.

The pulse spectral phase is found by use of the same method as that illustrated in Fig. 1, which enables the retrieval of the phase from the time-domain fringes. 


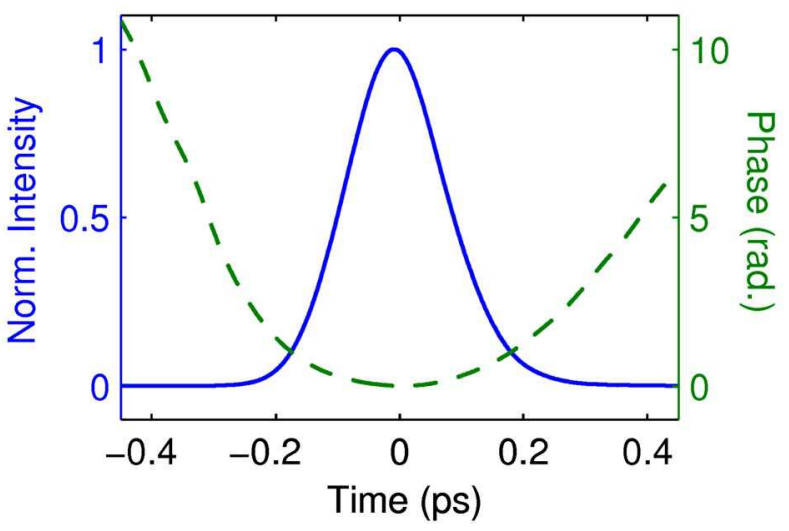

Fig. 7. (Color online) The reconstructed intensity (solid curve) and phase (dashed curves) in the time domain of the same mid-IR pulse as in Fig. 6 measured by TEA SPIDER. The reconstruction is an average over 1000 laser shots, with a shear frequency of $0.5 \mathrm{THz}$.

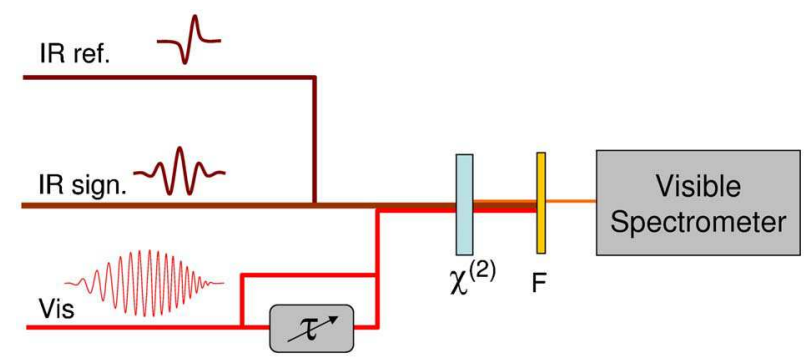

Fig. 8. (Color online) Experimental setup for performing mid-IR spectral interferometry and TEA SPIDER characterization of the mid-IR reference pulse by use of a visible spectrometer and CCD camera.

Figure 7 shows the mid-IR pulse intensity $|\mathcal{E}(t)|^{2}$ and phase $\phi(t)$ in time domain reconstructed from the above procedure.

Finally, we note that TEA SPIDER can be associated with spectral interferometry in the setup shown in Fig. 8, which provides a convenient method for both implementing spectral interferometry and characterizing the reference pulse in situ. Indeed, when the signal beam is blocked, the reference pulse can be characterized by use of TEA SPIDER as discussed above. Afterwards, the signal beam is unblocked, and one arm of the interferometer is blocked or the interferometer bypassed, turning the setup into a CPU mid-IR spectrometer as in Fig. 2(d), with a CCD camera rather than an HgCdTe detector array.

\section{CONCLUSION}

To summarize, there are many methods available for characterizing mid-IR femtosecond pulses. Deciding which method is the best depends on the associated experiment as well as on the laser source.

In terms of the laser source, the main points are the repetition rate and the availability of additional pulses. For slower repetition rates of about $10 \mathrm{~Hz}$ or less, single shot methods such as SPIDER or CPU ZAP SPIDER are preferable to avoid low-speed scans. In contrast, high-rate sources such as oscillators make time-domain methods such as electro-optic sampling or time-domain interferometry quite practical. Furthermore, fast-scanning methods with two oscillators at different repetition rates allow especially high data-rate characterization [64-66]. For limited pulse availability, such as at a free-electron laser where other synchronised pulses are difficult to produce, a self-referenced method such as those discussed in Section 3 is needed.

The choice of pulse characterization also depends on the requirements of the experiment. For example, the $\mathrm{CE}$ phase, which is important for some high-field experiments, cannot be determined by the methods discussed in Sections 3 and 4 . In contrast, electro-optic sampling directly accesses the electric field and hence the CE phase. Interferometric methods are also suitable for measuring the $\mathrm{CE}$ phase if they use a phase-stabilized reference pulse such as might be produced by optical rectification.

When sensitivity is important, e.g., for measuring tiny echo signals encountered in multidimensional spectroscopy, interferometric methods such as those discussed in Subsection 2.B are usually appropriate due to the enhanced sensitivity resulting from homodyning with the reference pulse (local oscillator). If one prefers to avoid using a costly, HgCdTe detector array with a low number of pixels, CPU methods such as those discussed in Section 4 represent a convenient approach in the case of a CPA laser source.

\section{ACKNOWLEDGMENTS}

We gratefully acknowledge Antigoni Alexandrou for her key software contributions to achieving a $1 \mathrm{kHz}$ data acquisition rate, and Xavier Solinas for contributing his electronics expertise. This research was supported by Agence Nationale de la Recherche (ANR-06-BLAN-0286) and by Conseil Général de l'Essonne (Astre 2006).

\section{REFERENCES}

1. R. M. Hochstrasser, "Two-dimensional spectroscopy at infrared and optical frequencies," Proc. Natl. Acad. Sci. U.S.A. 104, 14190-14196 (2007).

2. R. N. Bracewell, The Fourier Transform and Its Applications, 3rd ed. (McGraw-Hill, 1999).

3. M. Takeda, H. Ina, and S. Kobayashi, "Fourier-transform method of fringe-pattern analysis for computer-based topography and interferometry," J. Opt. Soc. Am. A 72, 156-160 (1982).

4. E. M. Kosik, A. S. Radunsky, I. A. Walmsley, and C. Dorrer, "Interferometric technique for measuring broadband ultrashort pulses at the sampling limit," Opt. Lett. 30, 326-328 (2005).

5. A. S. Wyatt, I. A. Walmsley, G. Stibenz, and G. Steinmeyer, "Sub-10 fs pulse characterization using spatially encoded arrangement for spectral phase interferometry for direct electric field reconstruction," Opt. Lett. 31, 1914-1916 (2006).

6. L. Lepetit, G. Cheriaux, and M. Joffre, "Linear techniques of phase measurement by femtosecond spectral interferometry for applications in spectroscopy," J. Opt. Soc. Am. B 12, 2467-2474 (1995).

7. C. Iaconis and I. A. Walmsley, "Spectral phase interferometry for direct electric-field reconstruction of ultrashort optical pulses," Opt. Lett. 23, 792-794 (1998).

8. J. R. Birge, R. Ell, and F. X. Kartner, "Two-dimensional spectral shearing interferometry for few-cycle pulse characterization," Opt. Lett. 31, 2063-2065 (2006).

9. A. Baltuska, M. Uiberacker, E. Goulielmakis, R. Kienberger, V. S. Yakovlev, T. Udem, T. W. Hänsch, and F. 
Krausz, "Phase-controlled amplification of few-cycle laser pulses," IEEE J. Sel. Top. Quantum Electron. 9, 972-989 (2003).

10. R. A. Kaindl, M. Wurm, K. Reimann, P. Hamm, A. M. Weiner, and M. Woerner, "Generation, shaping, and characterization of intense femtosecond pulses tunable from 3 to $20 \mu \mathrm{m}$," J. Opt. Soc. Am. B 17, 2086-2094 (2000).

11. C. Ventalon, J. M. Fraser, J. P. Likforman, D. M. Villeneuve, P. B. Corkum, and M. Joffre, "Generation and complete characterization of intense mid-infrared ultrashort pulses," J. Opt. Soc. Am. B 23, 332-340 (2006).

12. A. Bonvalet, M. Joffre, J. L. Martin, and A. Migus, "Generation of ultrabroadband femtosecond pulses in the mid-infrared by optical rectification of $15 \mathrm{fs}$ light pulses at $100 \mathrm{MHz}$ repetition rate," Appl. Phys. Lett. 67, 2907-2909 (1995).

13. Q. Wu and X.-C. Zhang, "Free-space electro-optics sampling of mid-infrared pulses," Appl. Phys. Lett. 71, 1285-1287 (1997).

14. J.-P. Likforman, M. Mehendale, D. M. Villeneuve, M. Joffre, and P. B. Corkum, "Conversion of high-power 15-fs visible pulses to the mid infrared," Opt. Lett. 26, 99-101 (2001)

15. C. Kübler, R. Huber, S. Tübel, and A. Leitenstorfer, "Ultrabroadband detection of multi-terahertz field transients with GaSe electro-optic sensors: Approaching the near infrared," Appl. Phys. Lett. 85, 3360-3362 (2004).

16. T. Zentgraf, R. Huber, N. C. Nielsen, D. S. Chemla, and R. A. Kaindl, "Ultrabroadband $50-130 \mathrm{THz}$ pulses generated via phase-matched difference frequency mixing in LiIO3," Opt. Express 15, 5775-5781 (2007).

17. K. J. Kubarych, M. Joffre, A. Moore, N. Belabas, and D. Jonas, "Mid-infrared electric field characterization using a visible charge-coupled-device-based spectrometer," Opt. Lett. 30, 1228-1230 (2005).

18. D. Grischkowsky, S. Keiding, M. Vanexter, and C. Fattinger, "Far-infrared time-domain spectroscopy with terahertz beams of dielectrics and semiconductors," J. Opt. Soc. Am. B 7, 2006-2015 (1990)

19. Q. Wu and X. C. Zhang, "Ultrafast electro-optic field sensors," Appl. Phys. Lett. 68, 1604-1606 (1996).

20. R. Huber, A. Brodschelm, F. Tauser, and A. Leitenstorfer, "Generation and field-resolved detection of femtosecond electromagnetic pulses tunable up to $41 \mathrm{THz}$," Appl. Phys. Lett. 76, 3191 (2000).

21. K. Liu, J. Z. Xu, and X. C. Zhang, "GaSe crystals for broadband terahertz wave detection," Appl. Phys. Lett. 85, 863-865 (2004).

22. T. Kampfrath, J. Notzold, and M. Wolf, "Sampling of broadband terahertz pulses with thick electro-optic crystals," Appl. Phys. Lett. 90, 231113 (2007).

23. E. Goulielmakis, M. Uiberacker, R. Kienberger, A Baltuska, V. Yakovlev, A. Scrinzi, T. Westerwalbesloh, U. Kleineberg, U. Heinzmann, M. Drescher, and F. Krausz, "Direct measurement of light waves," Science 305, 1267-1269 (2004).

24. N. Belabas and M. Joffre, "Visible-infrared twodimensional Fourier-transform spectroscopy," Opt. Lett. 27, 2043-2045 (2002).

25. N. Belabas, J. P. Likforman, L. Canioni, B. Bousquet, and M. Joffre, "Coherent broadband pulse shaping in the mid infrared," Opt. Lett. 26, 743-745 (2001).

26. A. Bonvalet, J. Nagle, V. Berger, A. Migus, J. L. Martin, and M. Joffre, "Femtosecond infrared emission resulting from coherent charge oscillations in quantum wells," Phys. Rev. Lett. 76, 4392-4395 (1996).

27. M. C. Asplund, M. T. Zanni, and R. M. Hochstrasser, "Two-dimensional infrared spectroscopy of peptides by phase-controlled femtosecond vibrational photon echoes," Proc. Natl. Acad. Sci. U.S.A. 97, 8219-8224 (2000).

28. S.-H. Shim, D. B. Strasfeld, E. C. Fulmer, and M. T. Zanni, "Femtosecond pulse shaping directly in the mid-IR using acousto-optic modulation," Opt. Lett. 31, 838-840 (2006).

29. S.-H. Shim, D. B. Strasfeld, and M. T. Zanni, "Generation and characterization of phase and amplitude shaped femtosecond mid-IR pulses," Opt. Express 14, 13120-13130 (2006).

30. D. N. Fittinghoff, J. L. Bowie, J. N. Sweetser, R. T. Jennings, M. A. Krumbugel, K. W. DeLong, R. Trebino, and I. A. Walmsley, "Measurement of the intensity and phase of ultraweak, ultrashort laser pulses," Opt. Lett. 21, 884-886 (1996).

31. C. Dorrer, N. Belabas, J. P. Likforman, and M. Joffre, "Spectral resolution and sampling issues in Fouriertransform spectral interferometry," J. Opt. Soc. Am. B 17, 1795-1802 (2000).

32. M. Khalil, N. Demirdoven, and A. Tokmakoff, "Obtaining absorptive line shapes in two-dimensional infrared vibrational correlation spectra," Phys. Rev. Lett. 90, 047401 (2003).

33. J. B. Asbury, T. Steinel, C. Stromberg, K. J. Gaffney, I. R. Piletic, A. Goun, and M. D. Fayer, "Hydrogen bond dynamics probed with ultrafast infrared heterodynedetected multidimensional vibrational stimulated echoes," Phys. Rev. Lett. 91, 237402 (2003).

34. V. Cervetto, J. Helbing, J. Bredenbeck, and P. Hamm, "Double-resonance versus pulsed Fourier transform twodimensional infrared spectroscopy: An experimental and theoretical comparison," J. Chem. Phys. 121, 5935-5942 (2004).

35. V. Wong and I. A. Walmsley, "Analysis of ultrashort pulseshape measurement using linear interferometers," Opt. Lett. 19, 287-289 (1994).

36. V. Wong and I. A. Walmsley, "Linear filter analysis of methods for ultrashort-pulse-shape measurements," J. Opt. Soc. Am. B 12, 1491-1499 (1995).

37. K. Naganuma, K. Mogi, and H. Yamada, "General method for ultrashort light-pulse chirp measurement," IEEE J. Quantum Electron. 25, 1225-1233 (1989).

38. R. Trebino, K. W. DeLong, D. N. Fittinghoff, J. N. Sweetser, M. A. Krumbugel, B. A. Richman, and D. J. Kane, "Measuring ultrashort laser pulses in the time-frequency domain using frequency-resolved optical gating," Rev. Sci. Instrum. 68, 3277-3295 (1997).

39. P. N. Butcher and D. Cotter, The Elements of Nonlinear Optics (Cambridge U. Press, 1990).

40. J. B. Asbury, T. Steinel, and M. D. Fayer, "Vibrational echo correlation spectroscopy probes of hydrogen bond dynamics in water and methanol," J. Lumin. 107, 271-286 (2004).

41. C. Ventalon, J. M. Fraser, and M. Joffre, "Time-domain interferometry for direct electric field reconstruction of mid-infrared femtosecond pulses," Opt. Lett. 28, 1826-1828 (2003).

42. J. Paye, M. Ramaswamy, J. G. Fujimoto, and E. P. Ippen, "Measurement of the amplitude and phase of ultrashort light-pulses from spectrally resolved autocorrelation," Opt. Lett. 18, 1946-1948 (1993).

43. G. Stibenz and G. Steinmeyer, "Interferometric frequencyresolved optical gating," Opt. Express 13, 2617-2626 (2005).

44. I. A. Roldan, I. G. Cormack, P. Loza-Alvarez, E. J. Gualda, and D. Artigas, "Ultrashort pulse characterisation with SHG collinear-FROG," Opt. Express 12, 1169-1178 (2004).

45. B. A. Richman, M. A. Krumbugel, and R. Trebino, "Temporal characterization of mid-IR free-electron-laser pulses by frequency-resolved optical gating," Opt. Lett. 22, 721-723 (1997).

46. T. Witte, K. L. Kompa, and M. Motzkus, "Femtosecond pulse shaping in the mid infrared by difference-frequency mixing," Appl. Phys. B 76, 467-471 (2003).

47. W. Wasilewski, P. Wasylczyk, and C. Radzewicz, "Femtosecond laser pulses measured with a photodiode FROG revisited," Appl. Phys. B 78, 589-592 (2004).

48. M. Tsubouchi and T. Momose, "Femtosecond pulse shaping in the mid-infrared generated by difference-frequency mixing: a simulation and experiment," J. Opt. Soc. Am. B 24, 1886-1900 (2007)

49. S. Yeremenko, A. Baltuska, F. de Haan, M. S. Pshenichnikov, and D. A. Wiersma, "Frequency-resolved pump-probe characterization of femtosecond infrared pulses," Opt. Lett. 27, 1171-1173 (2002). 
50. D. T. Reid, P. Loza-Alvarez, C. T. A. Brown, T. Beddard, and W. Sibbett, "Amplitude and phase measurement of midinfrared femtosecond pulses by using cross-correlation frequency-resolved optical gating," Opt. Lett. 25, 1478-1480 (2000).

51. J. A. Gruetzmacher and N. F. Scherer, "Few-cycle midinfrared pulse generation, characterization, and coherent propagation in optically dense media," Rev. Sci. Instrum. 73, 2227-2236 (2002).

52. H.-S. Tan and W. S. Warren, "Mid infrared pulse shaping by optical parametric amplification and its application to optical free induction decay measurement," Opt. Express 11, 1021-1028 (2003).

53. P. R. Griffiths and J. A. de Haseth, "Fourier transform infrared spectrometry," Chemical Analysis (Wiley, 1986), Vol. 83.

54. C. Dorrer, P. Londero, and I. A. Walmsley, "Homodyne detection in spectral phase interferometry for direct electric-field reconstruction," Opt. Lett. 26, 1510-1512 (2001).

55. A. Monmayrant, M. Joffre, T. Oksenhendler, R. Herzog, D. Kaplan, and P. Tournois, "Time-domain interferometry for direct electric-field reconstruction by use of an acoustooptic programmable filter and a two-photon detector," Opt. Lett. 28, 278-280 (2003).

56. M. Hirasawa, N. Nakagawa, K. Yamamoto, R. Morita, H. Shigekawa, and M. Yamashita, "Sensitivity improvement of spectral phase interferometry for direct electric-field reconstruction for the characterization of low-intensity femtosecond pulses," Appl. Phys. B 74, S225-S229 (2002).

57. P. Baum, S. Lochbrunner, and E. Riedle, "Zero-additionalphase SPIDER: full characterization of visible and sub-20fs ultraviolet pulses," Opt. Lett. 29, 210-212 (2004).
58. P. Baum, S. Lochbrunner, and E. Riedle, "Tunable sub-10-fs ultraviolet pulses generated by achromatic frequency doubling," Opt. Lett. 29, 1686-1688 (2004).

59. P. Baum and E. Riedle, "Design and calibration of zeroadditional-phase SPIDER," J. Opt. Soc. Am. B 22, 1875-1883 (2005).

60. M. J. Nee, R. McCanne, K. J. Kubarych, and M. Joffre, "Two-dimensional infrared spectroscopy detected by chirped pulse upconversion," Opt. Lett. 32, 713-715 (2007).

61. J. Treuffet, K. J. Kubarych, J.-C. Lambry, E. Pilet, J.-B. Masson, J.-L. Martin, M. H. Vos, M. Joffre, and A Alexandrou, "Direct observation of ligand transfer and bond formation in cytochrome c oxidase by using midinfrared chirped-pulse upconversion," Proc. Natl. Acad. Sci. U.S.A. 104, 15705-15710 (2007).

62. E. J. Heilweil, "Ultrashort-pulse multichannel infrared spectroscopy using broadband frequency conversion in LiIO3," Opt. Lett. 14, 551 (1989).

63. M. E. DeCamp and A. Tokmakoff, "Upconversion multichannel infrared spectrometer," Opt. Lett. 30, 1818-1820 (2005).

64. F. Keilmann, C. Gohle, and R. Holzwarth, "Time-domain mid-infrared frequency-comb spectrometer," Opt. Lett. 29, 1542-1544 (2004)

65. A. Bartels, A. Thoma, C. Janke, T. Dekorsy, A. Dreyhaupt, S. Winnerl, and M. Helm, "High-resolution $\mathrm{THz}$ spectrometer with $\mathrm{kHz}$ scan rates," Opt. Express 14, 430-437 (2006).

66. A. Bartels, R. Cerna, C. Kistner, A. Thoma, F. Hudert, C. Janke, and T. Dekorsy, "Ultrafast time-domain spectroscopy based on high-speed asynchronous optical sampling," Rev. Sci. Instrum. 78, 035107 (2007). 